\title{
Interference Cancellation in Respiratory Sounds via a Multiresolution Joint Time-Delay and Signal-Estimation Scheme
}

\author{
Sonia Charleston, Mahmood R. Azimi-Sadjadi,* Senior Member, IEEE, and Ramon González-Camarena
}

\begin{abstract}
This paper is concerned with the problem of cancellation of heart sounds from the acquired respiratory sounds using a new joint time-delay and signal-estimation (JTDSE) procedure. Multiresolution discrete wavelet transform (DWT) is first applied to decompose the signals into several subbands. To accurately separate the heart sounds from the acquired respiratory sounds, time-delay estimation (TDE) is performed iteratively in each subband using two adaptation mechanisms that minimize the sum of squared errors between these signals. The time delay is updated using a nonlinear adaptation, namely the Levenberg-Marquardt (LM) algorithm, while the function of the other adaptive system-which uses the block fast transversal filter (BFTF)—is to minimize the mean squared error between the outputs of the delay estimator and the adaptive filter. The proposed methodology possesses a number of key benefits such as the incorporation of multiple complementary information at different subbands, robustness in presence of noise, and accuracy in TDE. The scheme is applied to several cases of simulated and actual respiratory sounds under different conditions and the results are compared with those of the standard adaptive filtering. The results showed the promise of the scheme for the TDE and subsequent interference cancellation.
\end{abstract}

Index Terms-Adaptive filtering, biomedical signal processing, wavelet decomposition.

\section{TERMINOLOGY}

Throughout this paper we refer to the following terminologies.

Collected signal

Acquired respiratory sound Refers to that section of the collected signal where the heart-contaminated respiratory sound is confined.

Interference signal Refers to the heart sounds inside the acquired respiratory sound.

Manuscript received October 10, 1996; revised March 1, 1997. Asterisk indicates corresponding author.

S. Charleston is with the Department of Electrical Engineering, Universidad Autonoma Metropolitana, Mexico City 09340 Mexico.

*M. R. Azimi-Sadjadi is with the Department of Electrical Engineering, Colorado State University, Fort Collins, CO 80523 USA.

R. González-Camarena is with the Department of Health Sciences, Universidad Autonoma Metropolitana, Mexico City 09340 Mexico.

Publisher Item Identifier S 0018-9294(97)06905-X
The following signals are defined for the adaptive filter

Reference signal Refers to the heart sounds, first or second heart sounds, selected from the respiratory-free sections of the collected signal.

Primary signal Describes the acquired respiratory sound.

\section{INTRODUCTION}

O INCE the invention of the stethoscope, respiratory sounds auscultation has been a clinical approach commonly employed to monitor the course of lung diseases [1]. During the last decade or so, several attempts have been made to avoid the subjectivity of the method and to characterize the sounds through a more quantitative analysis. The sounds analysis has established the necessity to eliminate the interference signals prior to the extraction of relevant features [2]. The acquired signals not only contain respiratory sounds, but also heart sounds, ambient, muscle contraction, and hair noises. Some of these interferences could be eliminated to some extent by using a sound-proof room and/or an adequate microphone placement. However, the heart sounds are unavoidable and sometimes represent severe disturbing interference; each heartbeat produces two major sounds, known as the first and second heart sounds. In addition, intersubject and intrasubject biological variability and the overlap between frequency contents of the heart and respiratory sounds are important factors that preclude the application of deterministic filtering schemes [2], [3]. Some researchers apply highpass filtering schemes to remove the low-frequency part of the respiratory sound spectrum. However, in view of the studies carried out by other researchers [3]-[5], highpass filtering for this application results in loss of important signal information. More recently, various adaptive filtering schemes have been proposed to eliminate this kind of interference [6]-[9]. Adaptive-based approaches are considered to be the most practical, as they do not require any a priori information about the signals. However, they have only been partially successful, as their performance generally depends on accurate time alignment of the reference and primary signals. In [6] the reference signal to the adaptive filter was generated by adding to the acquired electrocardiographic (ECG) signal a delayed version of itself. The approach cannot follow the time variations between the first and second heart sounds or alterations in the 
cardiac frequency present in the acquired respiratory signal, because the distance between the original ECG and its delayed version is kept constant throughout the process. To avoid the acquisition of an additional signal, in [7] an elaborate scheme was applied to get the reference from the collected signal. The idea was to produce a spike whenever a heart sound occurred in the acquired respiratory signal through a combination of lowpass filtering and squared operation. The experimental results showed low to moderate heart sounds reduction [7]. More recently, the analysis of respiratory sounds on dogs was performed during anesthesia where at least two microphones were used and a strain gauge monitored the inspiration/expiration/rest periods [8]. The adaptive heart sounds cancellation was achieved using the phonocardiogram (PCG) signal present in the rest periods assuming that the neighboring PCG beats were correlated. The reference signals were formed with the PCG beats just before inhalation and just after exhalation periods. The location of the PCG signals in the respiratory section was determined by the cross-correlation method. It was observed that even after the adaptive filtering, PCG interference posed some problems, especially in the lower-frequency range. In [9] in order to avoid the time-delay alignment procedure the reduced-order Kalman filter (ROKF) was applied. To facilitate the estimation of the respiratory sounds, an autoregressive (AR) model was fitted to the heart signal information present in the segments of the collected signal, which are free of respiratory sounds. The state-space equations necessary for the ROKF were then established, considering the respiratory sound as a colored additive process in the observation equation. It was shown that the ROKF method produced heart sounds estimates which preserved the morphology of the original heart sounds. However, the drawbacks of the ROKF approach lie in the necessity to establish a model for the heart and respiratory sounds and the structural and computational complexity of the algorithm.

The study of the aforementioned schemes, as well as the closer understanding of the original problem, led to the conclusion that the performance of any time-domain cancellation scheme relies strongly on the accurate estimation of the time delay between the reference and primary signals. Consequently, in this paper the heart sounds interference cancellation is accomplished in two steps. The first step deals with the timedelay estimation (TDE) between the two sequences while the second step deals with the interference cancellation. TDE is needed not only for interference-cancellation type problems, but also for source localization, direction of arrival estimation, etc., [10], [11]. Several methods have been developed for TDE, but the problem is still a subject of extensive research, since delay estimation becomes very difficult in real-life situations. In this paper a new joint time delay and signal estimation (JTDSE) procedure is proposed using the dyadic multiresolution analysis through the discrete wavelet transform (DWT) [12]-[14]. The proposed methodology possesses a number of key benefits such as the incorporation of multiple complementary information at different subbands, robustness in presence of noise, and a validation procedure for the estimated delays.

The organization of this paper is as follows. Section II describes the modeling process of the acquired sounds in the context of time delay and signal estimation. Section III details on the fundamental idea and assumptions of the JTDSE scheme. The adaptation schemes for the TDE using the Levenberg-Marquardt (LM) algorithm [15] and the adaptive signal separation using the block fast transversal filter (BFTF) algorithm [16] are also briefly described in this section. The scheme is then applied to several cases of simulated and actual respiratory sounds in order to test its performance under different conditions and for a variable number of heart sound component scenarios. The results presented in Section IV show the promise of the proposed scheme for the TDE and subsequent interference cancellation.

\section{Modeling of the Acquired Respiratory Signal}

Let us consider the following model for the acquired respiratory signal, $z(n)$, which consists of multiple heart sounds, respiratory sound, and the background noise, i.e.,

$$
\begin{aligned}
z(n)= & \sum_{i=1}^{M_{1}} y_{1 i}\left(n-\Delta_{1 i}\right)+\sum_{j=1}^{M_{2}} y_{2 j}\left(n-\Delta_{2 j}\right) \\
& +v(n)+b(n), \quad n=0,1, \cdots, N
\end{aligned}
$$

where the first and second terms represent the effects of $M_{1}$ first heart sounds and $M_{2}$ second heart sounds with unknown time delays $\Delta_{1 i}$ and $\Delta_{2 j}$, respectively, $v(n)$ is the respiratory sound and $b(n)$ represents the effects of additive measurement noise. The first and second interference heart sounds $y_{1 i}(n)$ and $y_{2 j}(n)$ are of finite duration and nonoverlapping. Thus, we can write

$$
y_{1 i}(n)=0 \text { for } n<\Delta_{1 i}, \quad n>\Delta_{1 i}+T_{1 i}
$$

with

$$
\begin{gathered}
T_{1 i}<\Delta_{1, i+1}-\Delta_{1 i} \\
y_{2 j}(n)=0 \text { for } n<\Delta_{2 j}, \quad n>\Delta_{2 j}+T_{2 j}
\end{gathered}
$$

with

$$
T_{2 j}<\Delta_{2, j+1}-\Delta_{2 j}
$$

where $T_{1 i}$ and $T_{2 j}$ are the corresponding durations. In addition, these signals represent the "distorted" or modified version of the reference heart sounds, i.e.,

$$
\begin{aligned}
& y_{1 i}(n)=h_{1 i r}(n) * x_{1 r}(n) \\
& y_{2 j}(n)=h_{2 j r}(n) * x_{2 r}(n)
\end{aligned}
$$

where $*$ refers to the convolution operation, $x_{1 r}(n)$ and $x_{2 r}(n)$ represent the first and second heart reference sounds, and $h_{1 i r}(n)$ and $h_{2 j r}(n)$ denote the impulse responses of the "unknown" systems representing the correlation between the reference heart sounds and the interference heart sounds.

The model in (1) is arrived at based upon three assumptions using the properties of the contributing signals. First, the interaction between the heart sounds and the respiratory sound is assumed to be additive. Second, since the two sounds are generated by independent sources, they are assumed to be uncorrelated [6], [7]. In addition, the relationship between the heart sounds outside and within the respiratory segment is expressed as in (2) [8], [9]. Now, considering these assumptions, 


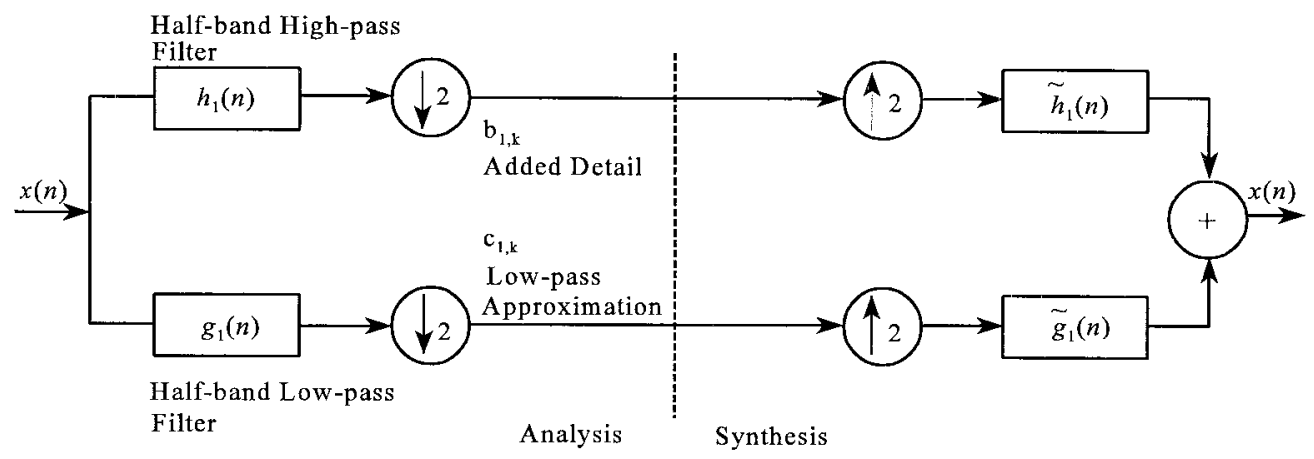

(a)
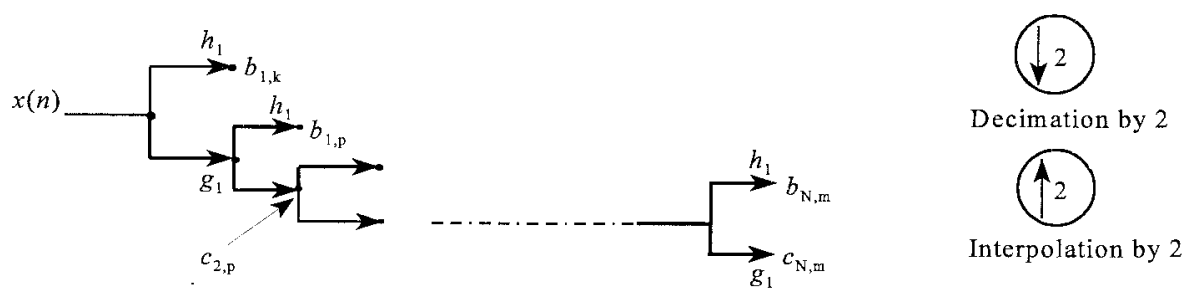

(b)

Fig. 1. (a) One-level multiresolution or subband analysis and synthesis. (b) Tree structure for DWT subband analysis containing $N$ levels.

the goal of the JTDSE method is to estimate the time delays $\Delta_{1 i}$ and $\Delta_{2 j}$ and then separate the corresponding heart sounds from the acquired respiratory signal. This is accomplished in two steps. In the first step the time delays associated with the heart sounds are estimated, i.e., the estimates of the positions of these interference signals are provided. The second step deals with the estimation of the interference and the subsequent cancellation process. The basic principle behind the proposed scheme is that the information about the time delays is common to all the information bearing subbands, hence, providing multiple "looks" of the same signal.

\section{Joint Time Delay AND Signal ESTIMATION (JTDSE) IN SUBbANDS}

\section{A. JTDSE Process}

The first step in the interference cancellation procedure is to determine the accurate position of each heart-sound component in the acquired respiratory signal. Once this is accomplished time alignment of the reference and the acquired signal and subsequent adaptive filtering for signal separation can be achieved in each subband. In this paper TDE is accomplished by using multiresolution or subband decomposition [12]-[14] of the reference signal and the acquired respiratory signal using the DWT. This decomposition permits the study of signals at different scales in a similar manner that one examines a geographical map, i.e., large scales correspond to global views while lower scales correspond to detailed views.

Let $x(n), n \in Z$ represent a square summable discretetime sequence. The multiresolution analysis of $x(n)$ can be interpreted as a successive decomposition of $x(n)$ in terms of its lower resolution and "detail" sequences. Using the subband decomposition scheme, a lower resolution and the "additional detail" sequences are generated by filtering $x(n)$ with a half- band lowpass filter, $g_{1}(n)$, and a half-band highpass filter, $h_{1}(n)$, respectively. These sequences can then be subsampled (decimated) by a factor of two, due to the Nyquist criterion, generating an increment in the scale. This procedure precisely forms one level of the multiresolution analysis as shown in Fig. 1(a). The lowpass approximation sequence can be decomposed subsequently as shown in Fig. 1(b). The filter bank has a tree structure as shown in Fig. 1(b) giving the well-known octave-band decomposition of the frequency axis, i.e., each level halves the width of the band and increases the frequency resolution by a factor of two. Note that due to subsampling by two, its time resolution is halved. The maximum resolution in the discrete-time case is determined by the original sampling rate.

Using the filter-bank approach for the DWT and assuming that the process of dividing the bands continues for $N$ levels, the final lowpass approximation sequence in Fig. 1(b) can be written as

$$
c_{N, k}=\sum_{n} x(n) g_{N}\left(2^{N} k-n\right)
$$

where $g_{N}(n)$ is expressed in terms of the impulse responses of the half-band lowpass filter as

$$
g_{N}(n)=\sum_{k} g_{N-1}(k) g_{1}(n-2 k) .
$$

Similarly, the "added details" sequences at different levels $j \in[1, N]$ are expressed in terms of the half-band highpass filter as

$$
b_{j, k}=\sum_{n} x(n) h_{j}\left(2^{j} k-n\right)
$$

where $h_{j}(n)$ is given by

$$
h_{j}(n)=\sum_{n} g_{j-1}(k) h_{1}(n-2 k), \quad j \in[2, N] .
$$




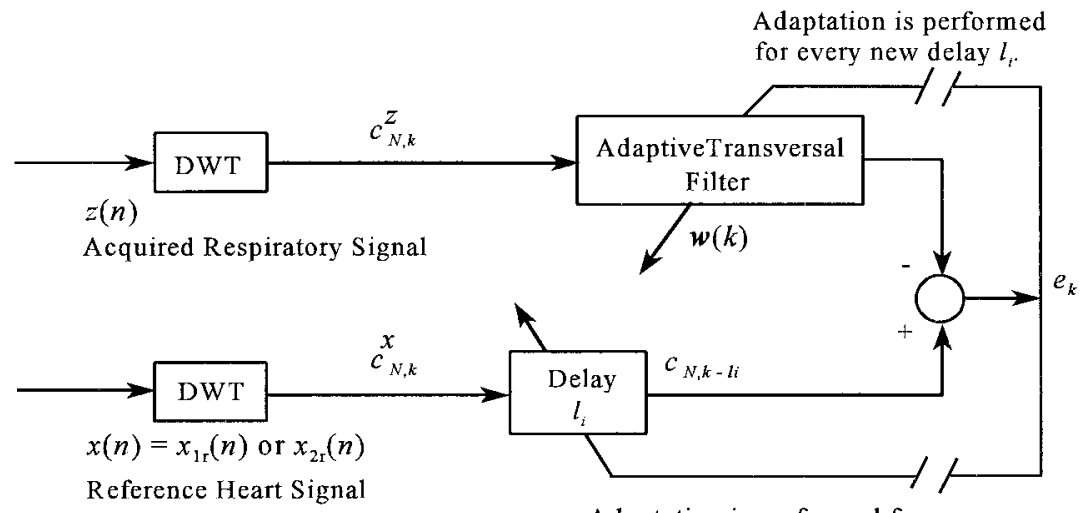

Adaptation is performed for every new optimal weight vector $\boldsymbol{w}(k)$.

Fig. 2. Joint time delay and signal estimation in subbands.

We assume that the filters are of finite-impulse response (FIR) type of order $L$ and the impulse responses and their shifted versions, by even shift, form orthogonal sets. It turns out that the lowpass and highpass filters are related by $h_{1}(n)=$ $(-1)^{n} g_{1}(L-1-n)$ as the modulation by $(-1)^{n}$ indeed transforms a lowpass to a highpass filter. Note that $L$ has to be even in order for the filters to form orthogonal sets.

The original sequence $x(n)$ can be recovered by upsampling by two followed by convolution with filters with impulse responses $\tilde{g}_{1}(n)$ and $\tilde{h}_{1}(n)$. It can easily be shown [12] that these are time-reversed versions of $g_{1}(n)$ and $h_{1}(n)$, respectively. The synthesis equation in terms of $c_{N, k}$ and $b_{j, k}, j \in[1, N]$ sequences and the corresponding impulse responses of the filters in the associated bands is given by

$$
x(n)=\sum_{j=1}^{N} \sum_{k} b_{j, k} \tilde{h}_{j}\left(n-2^{j} k\right)+\sum_{k} c_{N, k} \tilde{g}_{N}\left(n-2^{N} k\right) .
$$

Note that the above DWT analysis and synthesis equations, (3)-(5), are obtained based upon the assumption that the signals are of infinite extent. In practice, however, only finiteextent signals are encountered, in which case, in order to avoid border problems, one assumes that the original signal is symmetric [13], [14].

Once the signals are decomposed, the time delays are estimated in each subband, iteratively using two adaptive mechanisms as shown in Fig. 2. This figure demonstrates the entire process in one subband at the $N$ th level, where $c_{N, k}^{x}$ and $c_{N, k}^{z}$ are the final lowpass approximations for $x(n)$, which represents a first or a second heart sound reference, and for the acquired respiratory signal, $z(n)$, respectively. A similar process can be applied to the added details $b_{j, k}^{x}$ and $b_{j, k}^{z}, j \in[1, N]$. The reference signal, $x(n)$, in Fig. 2 is the first, $x_{1 r}(n)$, or the second, $x_{2 r}(n)$, heart sound sequences, manually selected from the nonbreathing part of the collected signal. The presence of measurement noise is inevitable in the chosen reference signal. However, due to fact that the ratio of the power of the heart sound to that of the measurement noise is very high, this does not impact the accuracy of the TDE. The reference is then shifted in the subband to different positions by $l_{i}$. The delay $l_{i}$ is updated using a nonlinear adaptation process to provide an estimate of $\Delta_{i}$, i.e., the time delay associated with the $i$ th heart sound. This is due to the property that the final lowpass approximation and the added detail approximations for the sequence $x(n-\Delta)$, with $\Delta=2^{N} l$ and $l$, an integer, are, respectively, $c_{N, k-l}^{x}$ and $b_{j, k-2^{N-j} l}^{x}, j \in[1, N]$. The updating rule for $l_{i}$ is based on the LM algorithm [15] as will be described later. For every new $l_{i}$, the function of the adaptive transversal filter is to modify its weights in order to generate an output signal which minimizes the mean squared error (MSE) at the output or maximizes the correlation or similarity between the outputs of the delay and the adaptive filter. To see this, let us for simplicity, assume that the acquired respiratory signal contains only one first heart sound and that the TDE is performed on the final lowpass approximated signals. In this case the output of the system (error signal) is

$$
e_{N, k}=c_{N, k-l_{i}}^{x_{1 r}}-w(k) * c_{N, k}^{z} .
$$

But, from (1) the final lowpass approximation of $z(n)$ is

$$
c_{N, k}^{z}=c_{N, k-\delta_{i}}^{y_{1}}+c_{N, k}^{v}+c_{N, k}^{b}
$$

where $\delta_{i}$ represents the actual time delay at level $N$ and $c_{N, k}^{y_{1}}, c_{N, k}^{v}$ and $c_{N, k}^{b}$ are the final lowpass approximations of $y_{1}(n), v(n)$ and $b(n)$, respectively. Thus, we have

$$
e_{N, k}=c_{N, k-l_{i}}^{x_{1 r}}-w(k) *\left[c_{N, k-\delta_{i}}^{y_{1}}+c_{N, k}^{v}+c_{N, k}^{b}\right] .
$$

Since $x_{1 r}(n)$ is assumed to be independent of the respiratory sound, $v(n)$, and the measurement noise, $b(n)$, when $l_{i}=\delta_{i}$ and, further, $w(k)$ captures the inverse model of the process $h_{1 i r}(n)$ in the relevant subband, maximum correlation would occur between the delayed reference $c_{N, k-l_{i}}^{x_{1 r}}$ and the output of the filter $w(k) * c_{N, k-\delta_{i}}^{y_{1}}$. It can easily be shown that this leads to the minimum of the mean squared value of $e_{N, k}$.

The original BFTF algorithm is used for updating the weights of the transversal filter as this method is inherently fast [16]. The process is repeated for several iterations for $l_{i}$ and the MSE curve is examined for its minima. The analysis of the minima of the MSE curve at each subband can be done using a simple thresholding operation. The positions at which these minima occur correspond to the locations where maximum correlations between the outputs of both adaptive 
systems are found, which in turn correspond to the estimates of the delays $\Delta_{i}$. Consequently, the time delays are computed once the complete MSE curve is obtained. To account for the time lag introduced by the FIR adaptive filter at these positions [17], the lag associated with the largest weight is subtracted from the delay estimate at each level. The final delay value is then calculated using $\Delta_{i}=2^{j}\left(l_{i}-q\right)$, where $j$ is the level index, $l_{i}$ is the estimated delay, and $q$ is the lag associated with the weight of the largest magnitude. The heart sounds are then separated from the acquired respiratory signal by means of a separate processing.

As a result of the filtering and decimation processes at each level, the signals are less correlated than those at the previous levels. This has two major benefits for our application. First, noise and other disturbances will be less prominent at the higher levels than in the original signal domain. Second, since the signals at the higher levels are also, less correlated, the estimate of the gradient in the LM algorithm will be less noisy. In addition, the lower levels (finer scales) offer increased accuracy in comparison with the higher levels. Thus, once the time delays in each subband are determined, the estimated delays can be validated against each other between the subbands and levels (if needed) due to the fact that in a dyadic multiresolution analysis the relationship between two consecutive levels is established by multiplication or division by two. Based on these principles, a decision rule was arrived at in order to select the time delays between two levels. If the number of minima is the same between the two levels, the time delay information is generally extracted from the lower level; otherwise, the time delay information is extracted from the next level.

\section{B. Time-Delay Adaptation Process}

As mentioned before, the delay $l_{i}$ is updated in each subband using a nonlinear adaptation process, namely, the LM method. Let us, for the sake of simplicity, use the lower-order approximation at level $N$ for the subsequent analysis. Note that a similar procedure can be applied to all the other subbands. If $c_{N, k-l_{i}}^{x}$ represents the output of the variable delay in Fig. 2, the index for minimization in this subband can be given by

$$
\xi_{N}=\sum_{k=1}^{m} e_{N, k}^{2}=\sum_{k=1}^{m}\left(c_{N, k-l_{i}}^{x}-\boldsymbol{w}_{k}^{t} c_{k}^{z}\right)^{2}
$$

where $w_{k}=\left[w_{0}(k) \cdots w_{M-1}(k)\right]^{t}$ and $c_{k}^{z}=\left[c_{N, k}^{z} \cdots\right.$ $\left.c_{N, k-M+1}^{z}\right]^{t}$ are the weight vector for the adaptive BFTF of order $M$ and its input vector at time $k$, respectively. As described before, for every new $l_{i}$, the minimization with respect to $\boldsymbol{w}_{k}$ is performed using the BFTF algorithm [16], which is explained later. Once the optimum weight vector for that specific $l_{i}$ is obtained, the procedure is repeated by updating $l_{i}$ using the LM algorithm [15]. For the time-delay adaptation, the goal is to iteratively find the necessary changes for $l_{i}$ that lead to the minimum of the cost function (9) using the following adaptation rule

$$
l_{i}(p)=l_{i}(p-1)+\Delta l_{i}
$$

where $p$ is the iteration index and $\Delta l_{i}$ is the updating step. The updating step $\Delta l_{i}$ can be generated using different nonlinear adaptation approaches such as the steepest descent method, which converges linearly and may be inefficient, particularly as the minimum is approached, or the Newtons method which converges quadratically provided that an adequate initial estimate of the unknown weight vector is available [15]. In this paper, the LM optimization algorithm [15] which is a nonlinear least squares-based method and provides a mean for interpolating between the Gauss-Newton and the steepestdescent steps is used. The algorithm possesses quadratic convergence close to a minimum, where it approximates the Gauss-Newton method. If the initial estimates are relatively poor, its convergence degenerates to the method of steepest descent.

Using the LM algorithm for TDE in each subband and defining $J \doteq\left[\nabla_{l_{i}} e_{k}\right]=\left(\begin{array}{ll}\nabla e_{1} & \nabla e_{2} \cdots \nabla e_{m}\end{array}\right)^{t}$, where $e_{k} \doteq$ $c_{N, k-l_{i}}^{x}-w_{k}^{t} c_{k}^{z}$, the following updating rule for $l_{i}$ can be given:

$$
\Delta l_{i}=\operatorname{Int}\left[\left(J^{t} J+\beta\right)^{-1} *\left(2 \sum_{\mathrm{k}} \mathrm{J}_{\mathrm{k}} *\left(\mathrm{c}_{\mathrm{N}, \mathrm{k}-\mathrm{l}_{\mathrm{i}}}^{\mathrm{x}}-\boldsymbol{w}_{\mathrm{k}}^{\mathrm{t}} c_{\mathrm{k}}^{\mathrm{z}}\right)\right)\right]
$$

where $\operatorname{Int}[\mathrm{x}]$ represents the integer part of $x$

$$
J_{k}=2 \frac{\partial c_{N, k-l_{i}}^{x}}{\partial l_{i}}=2^{N+1} \sum_{n} x(n) g_{N}^{\prime}\left(2^{N}\left(k-l_{i}\right)-n\right)
$$

and $g_{N}^{\prime}(\cdot)$ is computed using the forward difference method [18]. The parameter, $\beta$, prevents the possibility of singularity of $\boldsymbol{J}^{t} \boldsymbol{J}+\beta$ term. If this parameter is close to zero, then the estimate is reduced to Gauss-Newton's method, but if $\beta$ is greater than $\boldsymbol{J}^{\boldsymbol{t}} \boldsymbol{J}$, then (11a) provides a steepest-descent step. The selection of the parameter $\beta$ in (11) is a crucial step in the application of the LM algorithm. A simple way for the selection of this parameter is to multiply it by some factor whenever a step would result in an increase of the cost function (9) or divide it by the same factor if the step produces a reduction of the cost function. However, instead of deciding whether a step is successful based on a simple decrease or increase of the cost function, a better approach is to decide whether the cost function at hand is adequately represented by a quadratic model, so that the convergence of the algorithm to a local minimum could be guaranteed. The criterion can be written as the ratio

$$
v=\frac{\xi_{N}\left(l_{i}\right)-\xi_{N}\left(l_{i}+\Delta l_{i}\right)}{\boldsymbol{J}^{t} \epsilon * \Delta l_{i}+1 / 2\left(\boldsymbol{J}^{t} \boldsymbol{J}+\beta\right) * \Delta l_{i}^{2}}
$$

where $\epsilon=\left[e_{1} \cdots e_{m}\right]^{t}$. The numerator represents the actual change in the value of the cost function (9), while the denominator represents the ideal change provided by the quadratic model of the cost function. A value of $v$ equal to or greater than one (1) represents an acceptable model representation, while a value less than one represents an inadequate model representation. The value of $\beta$ is not modified if $v$ is inside the admissible modeling region established between 0.25 and 0.75 [15]. However, outside this interval, the strategy is to select an initial value of $\beta$ and then increase (or decrease) it until the acceptable interval of $v$ is reached. 


\section{Weight Adaptation Using the BFTF}

The second adaptive process for updating the weights of the transversal filter uses the BFTF scheme [16]. This method is selected over all other adaptive schemes because of its unique features which include convergence speed, low computational requirements, and reduced round-off error effects. In general, adaptive schemes are divided in two classes: methods that update the weights per sample of the desired and reference signals, and those that make such adaptation once per block of data. The BFTF algorithm is computationally very efficient compared with other least squares (LS)-based schemes. In this method, a block-LS cost function is minimized in every block independently. As a result, the algorithm guarantees a locally optimal solution in each block. Let us start with a single data block, the BFTF approach determines the weight vector $\boldsymbol{w}_{M, L, k}$ which minimizes the block LS cost function

$$
\xi_{M, L, k}=\sum_{m=k-L+1}^{k}\left(c_{N, m-l_{i}}^{x}-w_{M, L, k} c_{m}^{z}\right)^{2}
$$

where $M$ is the order of the transversal filter vector, $L$ specifies the data block length, $k$ is the highest time index of the block, $l_{i}$ is the estimated delay, and $c_{m}^{z}$ is defined as before. Note that $\boldsymbol{w}_{M, L, k}$ is the same weight vector as $\boldsymbol{w}_{k}$ in (9). However, in this section indexes $M$ and $L$ are added to indicate dependency on the choice of the filter order and block size. Additionally, $\xi_{M, L, k}$ represents the same index for minimization defined over one block of data. Now, let us define the $L \times 1$ desired and input block vectors as $\boldsymbol{c}_{L, k-l_{i}}^{x} \doteq\left[c_{N, k-l_{i}}^{x} \cdots c_{N, k-l_{i}-L+1}^{x}\right]^{t}$ and $c_{L, k}^{z} \doteq\left[c_{N, k}^{z} \cdots c_{N, k-L+1}^{z}\right]^{t}$, respectively, and the $L \times M$ aggregate data matrix $\boldsymbol{C}_{M, L, k}^{z}$ as

$$
C_{M, L, k}^{z} \doteq\left[\boldsymbol{c}_{L, k}^{z}, \cdots, \boldsymbol{c}_{L, k-M+1}^{z}\right]
$$

With the error vector defined as $\epsilon_{M, L, k} \doteq \boldsymbol{c}_{L, k-l_{i}}^{x}$ $\boldsymbol{C}_{M, L, k}^{z} \boldsymbol{w}_{M, L, k}^{t}$, (13) can be rewritten as

$$
\xi_{M, L, k}=\epsilon_{M, L, k}^{t} \epsilon_{M, L, k} .
$$

In order for (15) to attain its minimum, the vector $\epsilon_{M, L, k}$ must be orthogonal to $C_{M, L, k}^{z}$, i.e., $\epsilon_{M, L, k}^{t} C_{M, L, k}^{z}=0$ (normal equation) [16]. The vector $\boldsymbol{w}_{M, L, k}$ that minimizes (15) is given by [16]

$$
\boldsymbol{w}_{M, L, k}=c_{L, k-l_{i}}^{x^{t}} C_{M, L, k}^{z}\left(C_{M, L, k}^{z t} C_{M, L, k}^{z}\right)^{\#}
$$

where \# denotes the generalized inverse which reduces to the standard inverse when the quantity involved is invertible. The goal of BFTF algorithm is to find a recursion for $w_{n+1, L, k}$ in terms of $w_{n, L, k}$, i.e., to update the weight vector of order $n+1$ in terms of the weight vector of order $n$. The algorithm consists of two steps. In the first step an order update rule is used intrablockwise to successively increase the order of the filter with the exact solution obtained at the $M$ th iteration. Once the final weight vector is obtained, the filtering is implemented on the data within the whole block in one pass. For details on the BFTF algorithm, see [16].

\section{RESUlTS AND Discussion}

The JTDSE in subbands described in the Section III was examined on two data sets. The goal of the first experiment was to test the JTDSE scheme on synthesized data with different heart-to-respiratory ratios (HRR's) and for variable number of heart sounds. The HRR is defined by $10 \log _{10}\left(\sigma_{y}^{2} / \sigma_{v}^{2}\right)$ where $\sigma_{y}^{2}$ and $\sigma_{v}^{2}$ are the interference signal and respiratory sound variances, respectively. In this study the locations of the heart sounds are known a priori. The second experiment, however, involved processing real data collected from a healthy subject with prominent heart sounds as well as the data from an asthmatic subject with obscured heart sounds conditions. The goal of this study was obviously to investigate the usefulness of our scheme for real-life situations.

\section{A. Results on Synthesized Data}

The first data set was generated using a segmented heart sound and an artificial respiratory sound. The segmented heart sound was obtained from a different subject, not included in the real cases of this paper. The reason was the low measurement noise power for this case. A variable number of heart sounds with different amplitudes and delays were buried in the artificial respiratory sound to generate different HRR conditions. The artificial respiratory signal was generated by filtering a Gaussian noise sequence, covering the same spectral range that is present in the real respiratory signals, and allowing spectral overlap between heart and respiratory frequency contents. In addition, to mimic the breathing-like shape of the respiratory signal, the filtered sequence was envelope modulated by a Hamming window in the time domain. This procedure generates the simulated respiratory sound for just one phase of the respiratory cycle. Note that the model for this synthesized respiratory signal can be obtained from (1) and (2) by setting $h_{1 i r}(n)=A_{1 i r} \delta(n)$ or $h_{2 j r}(n)=$ $A_{2 j r} \delta(n)$, hence, in this case the effects of distortion are ignored. In addition, with the exception of one case, the measurement noise $b(n)$ is zero since it does not present a dominant source of interference to impact the accuracy of the TDE. Fig. 3(a) shows the artificial respiratory signal and Fig. 3(b) shows the segmented second heart sound to be buried in the respiratory signal. Five synthesized cases were generated with two or four buried heart sounds and with the HRR's of -13.5 and $-19.5 \mathrm{~dB}$. These values are selected as they can be found in real-life situations and, moreover, provide difficult conditions for the detection of the heart sounds. Table I presents all the cases and their corresponding HRR's, together with the true and the estimated values of the delays. Fig. 3(c) and 3(d) presents the two synthesized signals corresponding to cases 1 and 3 in Table I, respectively. The second column in Table I shows the type of the heart signal inserted. The third column gives the HRR and respiratory-to-heart ratio (RHR) values for the cases generated. The numbers in the fourth and fifth columns represent the true and estimated time delays using the JTDSE- and the conventional cross-correlationbased methods, respectively. In all these synthesized data the measurement noise $b(n)$ was equal to zero, except in the first case in Table I, where a heart-to-measurement noise ratio of 
(a)

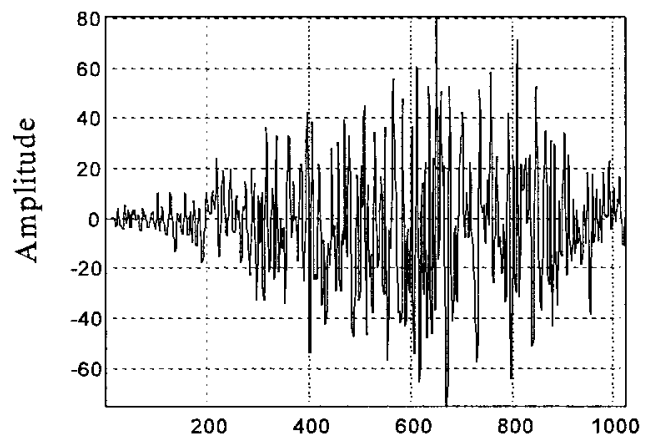

(c)

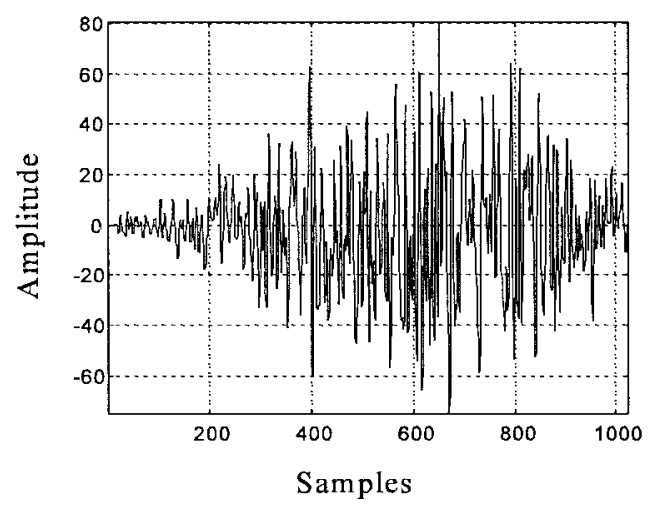

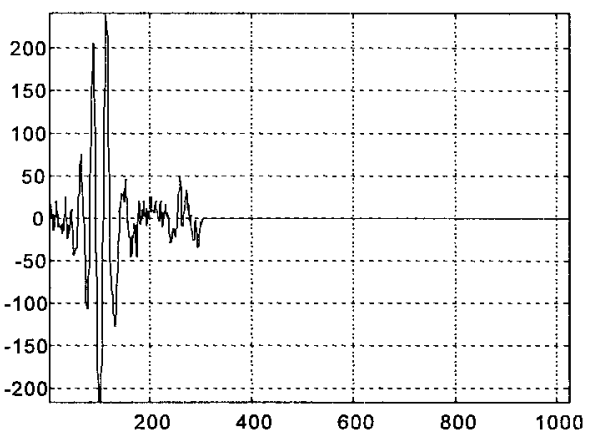

(b)

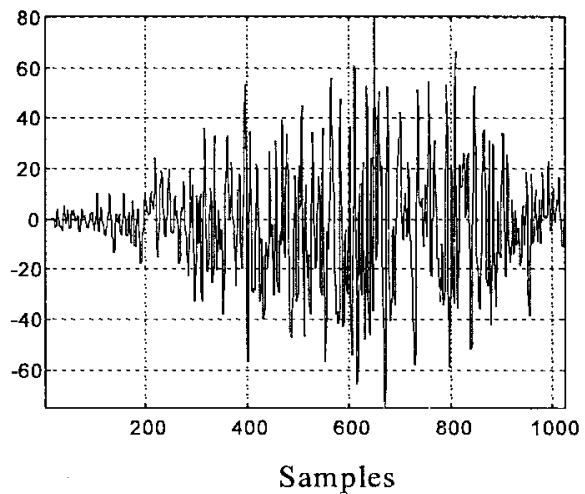

(d)

Fig. 3. (a) Artificial respiratory signal $v(n)$, (b) segmented second heart sound with measurement noise $x(n)+b(n)$, (c) synthesized signal $z(n)$ with HRR $=-13.5 \mathrm{~dB}$ (case 1 in Table I), and (d) synthesized signal $z(n)$ with HRR $=-19.5 \mathrm{~dB}$ (case 3 in Table I).

TABLE I

True AND Estimated Delays for Different HRR (RHR) VAlues

\begin{tabular}{|c|c|c|c|c|c|c|}
\hline Case & Heart Sound & HRR (RHR) (dB) & Time Delay & Estimates JTDSE Method & Time Delays & Estimates Conventional Method \\
\hline \multirow{2}{*}{1} & \multirow{2}{*}{ Second } & \multirow{2}{*}{$-13.5(13.5)$} & True: & 280,680 & True: & 280,680 \\
\hline & & & Estimated: & 280,672 & Estimated: & $263,383,465,590,679$ \\
\hline \multirow{2}{*}{2} & \multirow{2}{*}{ Second } & \multirow{2}{*}{$-13.5(13.5)$} & True: & 280,680 & True: & 280,680 \\
\hline & & & Estimated: & 280,680 & Estimated: & $280,375,465,556,678$ \\
\hline \multirow{2}{*}{3} & \multirow{2}{*}{ Second } & \multirow{2}{*}{$-19.5(19.5)$} & True: & 280,680 & True: & $280,424,544,680$ \\
\hline & & & Estimated: & 280,680 & Estimated: & $263,374,466,556,651,759$ \\
\hline \multirow{2}{*}{4} & \multirow{2}{*}{ Second } & \multirow{2}{*}{$-13.5(13.5)$} & True: & $280,390,570,680$ & True: & $280,390,570,680$ \\
\hline & & & Estimated: & $280,392,568,680$ & Estimated: & $263,383,556$ \\
\hline \multirow[b]{2}{*}{5} & \multirow{2}{*}{$\begin{array}{l}\text { First and } \\
\text { Second }\end{array}$} & \multirow[b]{2}{*}{$-13.5(13.5)$} & True: & 280,680 & True: & 280,680 \\
\hline & & & Estimated: & 280,680 & Estimated: & $280,234,374,516,556,650,680$ \\
\hline
\end{tabular}

$7 \mathrm{~dB}$ was used, according to the power of the noise present in the collected real data. The heart sound $x(n)$ and the synthesized signal $z(n)$ were then applied to the system in Fig. 2 with an eighth-order adaptive transversal filter. The dyadic decomposition was carried out for three levels using the Daubechies wavelets [12]. Precautions were taken to avoid edge effects in computing DWT coefficients for finite length sequences by considering symmetric extension of the original sequences.

Once the MSE curve in each subband was generated, the minima selection was performed through a thresholding operation that is MSE-curve dependent. The thresholding operation considers the MSE-curve values and their probabilities through the use of the MSE-curve histogram. It is assumed that the prominent MSE minima have lower probability in comparison with the rest of the MSE-curve values. The operation requires ordering the MSE-curve values from the global minimum to the global maximum to initialize a vector associated with the global minimum or the first minimum. A simple distance-based aggregation rule helps to decide if the next MSE-curve value in the ordered list is close to the first minimum or represents a different minimum in the MSE curve. Each time that a new minimum value is added to the vector, a decision about whether or not to include another minimum in the vector is made, based on the probability computation from the MSEcurve histogram. The multiple time-delay estimates generated in the subbands can then be validated to arrive at the final estimates. Table II provides the estimated time delays in all the subbands for the second and third cases in Table I. As can be seen from the results in this table, a simple decision 
(a)

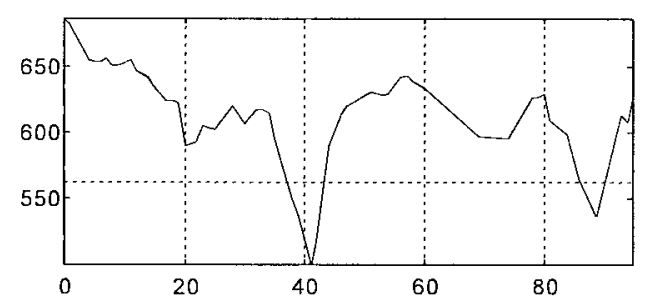

(b)

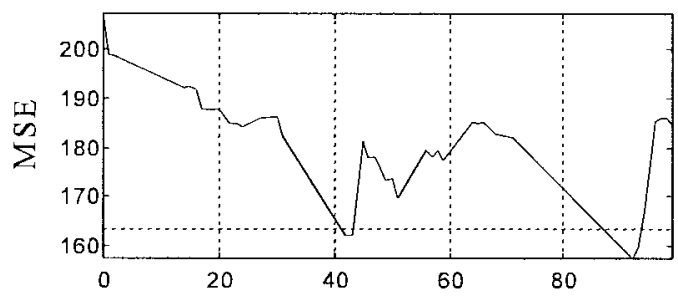

(c)

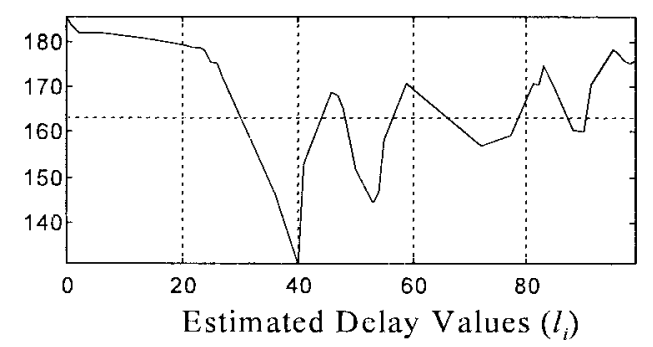

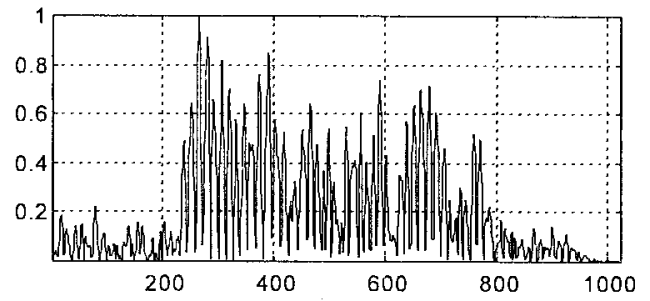

(d)

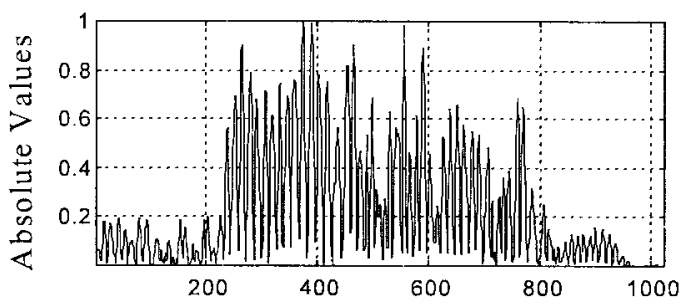

(e)

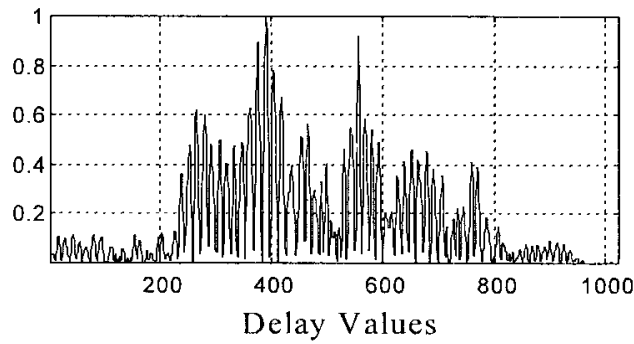

Fig. 4. (a)-(c) MSE curves for the JTDSE scheme at the third level of decomposition for cases 1, 3, and 4 of Table I, respectively. The horizontal dashed line in the MSE curves indicates the threshold. (d)-(f) Absolute value of the cross-correlation result between $x(n)$ and $z(n)$ for case 1,3 , and 4 of Table I.

rule can be devised to select the common time-delay estimates according to their frequency of appearance in all the bands. Our empirical studies indicate that subbands four and five provide consistently valid estimates. Consequently, for all other cases the delay estimates were computed based upon these two bands only.

Fig. 4 presents the results for the first, third, and fourth cases of the Table I. The actual delays of the heart sounds components in the first and third cases were 280 and 680, while the HRR of the third case, $-19.5 \mathrm{~dB}$, was lower than the first one, $-13.5 \mathrm{~dB}$. For the fourth case the actual delays were $280,390,570$, and 680 as is shown in Table I. In all these cases, the second heart sound was used both as the interference and reference signal to the filter. Fig. 4(a)-(c) presents the MSE curves for these cases at the third level of the decomposition and for the fourth subband. The first curve, Fig. 4(a), presents well-defined minima at two positions $l_{1}=39$ and $l_{2}=86$. For the third case, even though the HRR is very low, it is possible to identify two prominent minima at $l_{1}=42$ and $l_{2}=92$. The estimated values of time delays were extracted from these MSE plots by applying the adaptive thresholding operation mentioned before. These values were then corrected by the delays caused by the largest weight of the transversal filter, $q_{1}=4$ and $q_{2}=2$ for the first case and $q_{1}=7$ and $q_{2}=7$ for the third case. The results were then multiplied by $2^{N}$, i.e., $\Delta_{i}=2^{N}\left(l_{i}-q\right)$, where $N=3$, in order to provide the estimates of $\Delta_{i}$ as given in Table I. For the first case, the second estimated
TABLE II

Estimated Delay for Several Subbands for (a) Second and (b) Third Cases of Table I

\begin{tabular}{c|c|c|c|c|c|c|c|c|c|c|c|c}
\hline Bands & \multicolumn{10}{|c}{ Estimated delay values } \\
\hline 1 & & 264 & & & & & 544 & & & 680 & & \\
\hline 2 & & & 280 & & 384 & & & 560 & & & & \\
\hline 3 & & & 280 & & & 432 & & & & & 696 & \\
\hline 4 & & & 280 & & & & & & & 680 & & \\
\hline 5 & & & 280 & & & & & & & 680 & & \\
\hline 6 & & & & 320 & & & & & & & & 752 \\
\hline 7 & 0 & & 280 & & & & & & 600 & 680 & & \\
\hline 8 & & & 280 & & & & & & & 680 & & \\
\hline
\end{tabular}

(a)

\begin{tabular}{c|c|c|c|c|c|c|c|c|c}
\hline Bands & \multicolumn{7}{|c}{ Estimated delay values } \\
\hline 1 & & & & & 464 & 544 & & & \\
\hline 2 & & & & & & & 560 & & \\
\hline 3 & & & 320 & 432 & & & & 640 & \\
\hline 4 & & 280 & & & & & & & 680 \\
\hline 5 & & 280 & & & & & & & 680 \\
\hline 6 & & & 320 & & & & & & \\
\hline 7 & 0 & 280 & & & & & & 600 & 680 \\
\hline 8 & & 280 & & & & & & & 680 \\
\hline
\end{tabular}

(b)

time delay is slightly perturbed from the true delays as seen in Table I, while for the third case, the estimated values are equal to the true values. Once the delays are estimated, time-alignment can be achieved and filtering carried out in 
(a)
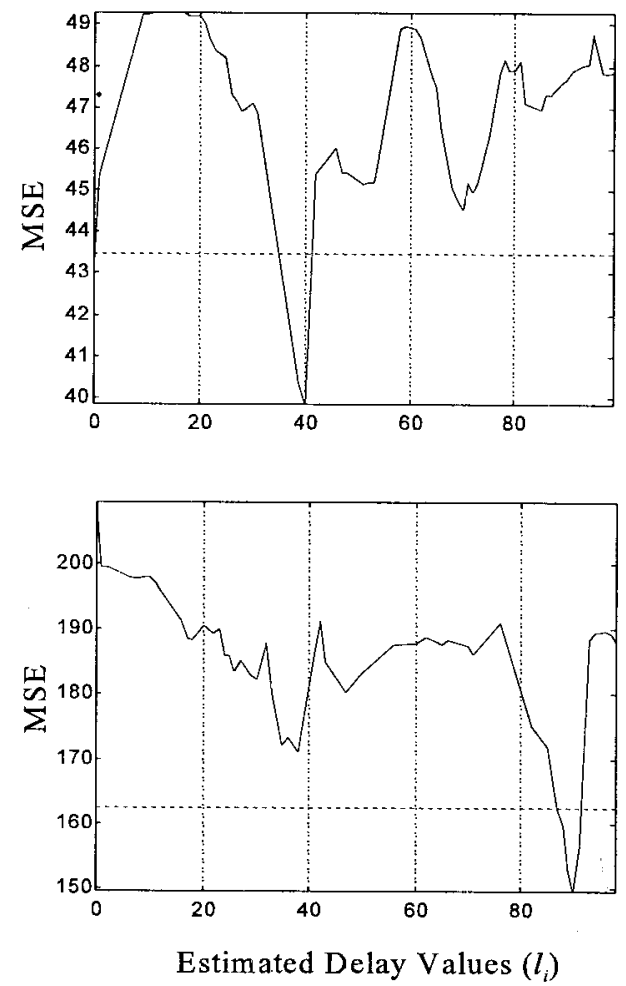

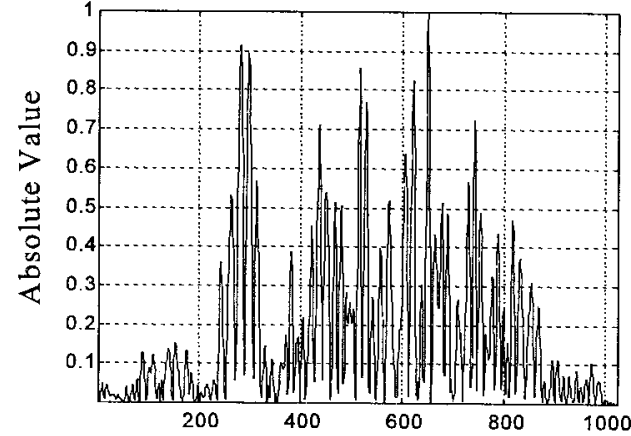

(c)

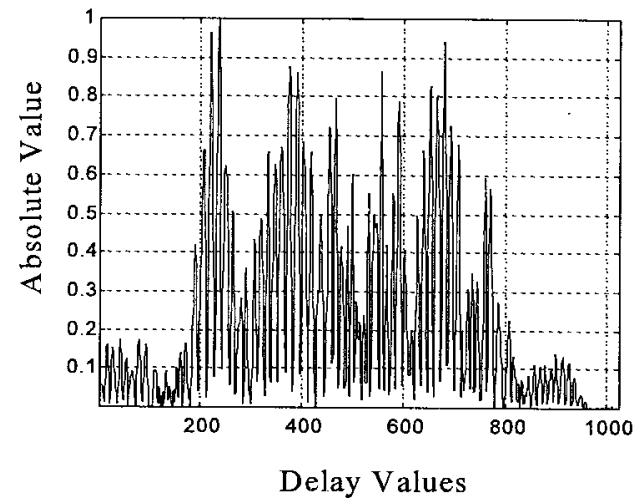

Fig. 5. Fifth case of Table I, containing a first and a second heart sound. MSE curve for JTDSE using (a) a first heart and (b) a second heart sound as the reference. The horizontal dashed line in the MSE curves indicates the threshold for minima selection. (c) Absolute value of the cross-correlation for a first heart sound reference and (d) cross-correlation result for a second heart sound reference.

the subband. The processed signal can then be reconstructed at the original signal scale. Fig. 4(d) and (f) presents the results of the cross-correlation between the reference and the synthesized signals, which is still widely used for timealignment purposes [8]. A thresholding procedure was applied to the normalized absolute value of the cross-correlations in Fig. 4(d) and (f). The delay values were estimated from these curves by thresholding the envelope of the normalized absolute cross-correlation. An adequate value for the threshold that worked satisfactorily for all the cases was fixed at 0.6; a value below this was considered to represent poor correlation. The estimated delays using this method are also given in Table I. As can be observed, the cross-correlation method provided a rather large number of spurious time-delay estimates. Even an adaptive threshold method for the cross-correlation function could generate several spurious time delay estimates.

The effectiveness of these methods was also tested when the time delays became close together, while the HRR was kept constant. Although this case does not typically occur in reallife situations, as there is always a time difference between the onset of the first and second heart sounds, the case is presented here to demonstrate the potential of the JTDSE method for separating close components. Fig. 4(c) presents the fourth case of Table I where four heart components are present in the synthesized signal. The MSE curve indicates the presence of four heart sound components through four welldefined minima. In this case, the JTDSE method generated accurate estimates of the positions of all the heart sound components inside the synthesized signal and the estimated values match very closely with the true values as seen in Table I. The cross-correlation method, on the other hand, failed as it provided three estimated delays and those estimates were not even correct.

The last case in Table I corresponds to a synthesized signal containing a first and a second heart sound components. Real examples of this kind will be presented in the next section. It is generally correct to assume that the first heart sound is more closely correlated with another first sound than a second heart sound as they are produced by the same cardiac source. A similar assumption can be made for the second heart sound. Consequently, in situations where multiple different heart sounds are present, two different reference signals must be used in the JTDSE method. Note that one can not use both the first and second heart sounds as the reference, as the time interval between them is generally variable. Fig. 5(a) and (b) shows the plots of the MSE curves for the JTDSE when a first and second heart sounds are used as a reference, respectively. Again, the curves exhibit well-defined minima at two different positions indicating the presence of two different heart sound components in the synthesized signal. The estimated delays at fourth subband are given in the last row of Table I. Fig. 5(b) and (d) shows the results of cross-correlation method which show estimated delays at 280, 516, and 650 for the first heart sound and 234, 374, 556, and 680 for the second heart sound.

\section{B. Results on Real Data}

The second data set consisted of real signals collected from a healthy subject and an asthmatic patient. This data was 
(a)

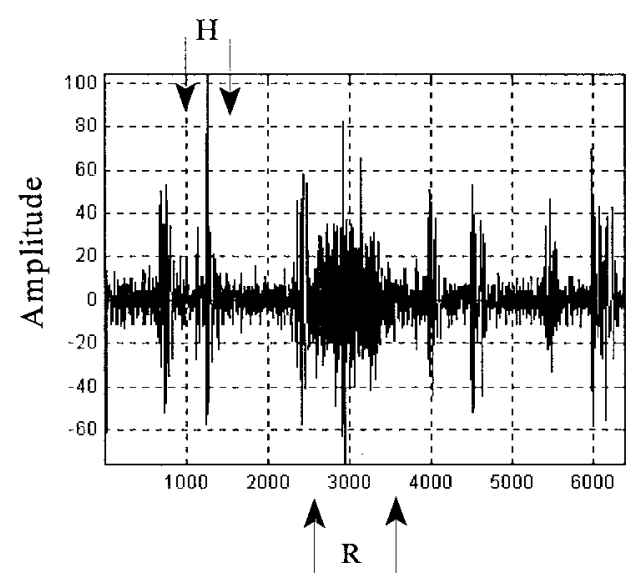

(c)

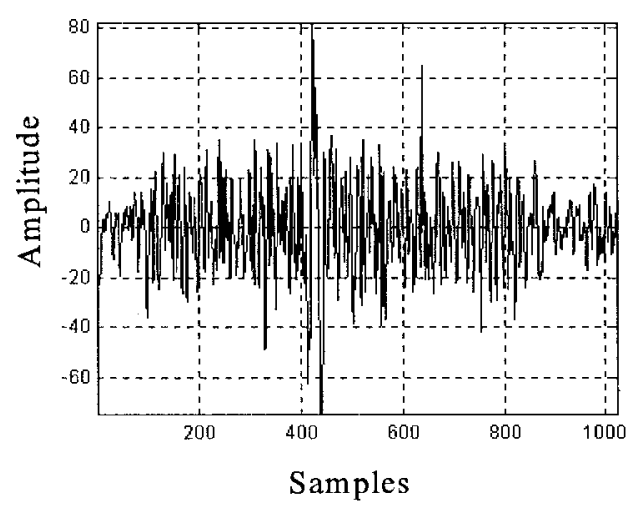

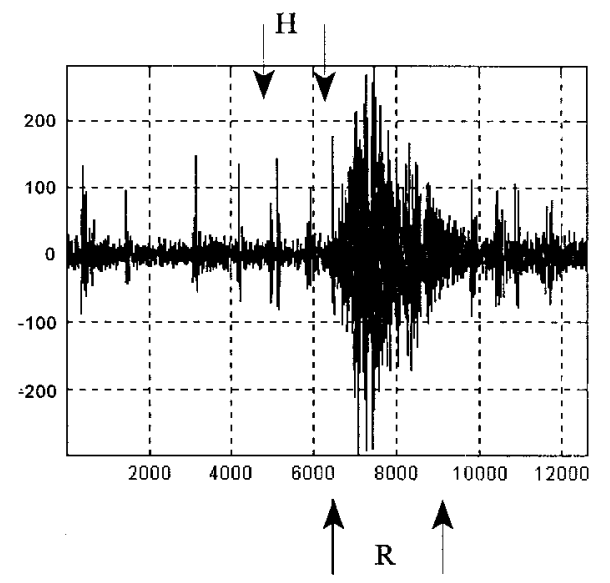

(b)

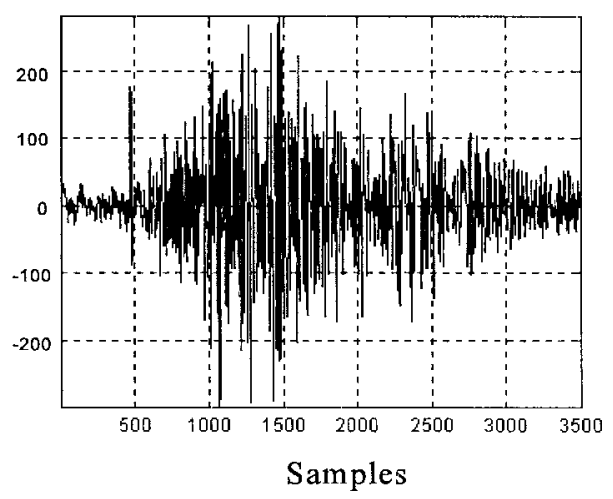

(d)

Fig. 6. (a) Collected signal from a healthy subject. (b) Collected signal from an asthmatic patient. (c) Manually selected acquired respiratory section $z(n)$ from the signal in (a). (d) Manually selected acquired respiratory section $z(n)$ from the signal in (b). The respiratory sections are identified $\mathbf{R}$ in (a) and (b) while $\mathbf{H}$ identifies the selected reference heart sounds.

acquired at trachea and cardiac apex levels and sampled at a rate of $4 \mathrm{KHz}$. Note that in all the experiments manual segmentation was performed in order to extract the acquired respiratory segments and the reference heart sounds. The reference signal was selected from those heart sounds close to (before or after) the acquired respiratory section. Although, it is possible to implement an automatic segmentation scheme based on signals like the air flow or changes in thoracic electrical impedance with the respiratory cycle [19], this was not implemented here as it is not related to the main thrust of the paper. Fig. 6(a) and (b) shows the collected signals including several heart sounds. It is possible to clearly see the only heart sound component inside the acquired respiratory section in the first case, as the HRR is higher than in the second case. This case was included in the testing in order to verify that under the high HRR condition the results of the JTDSE and cross-correlation methods are identical. In contrast to the first case, the second case, as shown in Fig. 6(b) and (d), is complicated due to several factors; such as low HRR, presence of multiple heart sound components, and variations in the cardiac frequency. Fig. 6(c) and (d) shows the manually extracted acquired respiratory sections for these cases.

In any real case, multiple first and second heart sounds may be present in the acquired respiratory signal. In addition, as pointed out before, the heart sounds outside and inside the acquired respiratory section are correlated. Consequently, for more accurate TDE, it is better to examine the results of both the first and second heart sounds as a reference for the JTDSE scheme. These heart sounds are manually extracted from the collected signals in Fig. 6(a) and (b) in sections that are close to the acquired respiratory section. The results of both heart sounds can then be combined for the filtering process. The heart-to-measurement noise ratio (HNR) was about $7 \mathrm{~dB}$ for the cases shown in Fig. 6(a) and (b). For the case in Fig. 6(c), the MSE curve is obtained using the JTDSE method with an eighth-order BFTF filter and the second heart sound as a reference is shown in Fig. 7(a). The corresponding MSE curve using the first heart sound as the reference gave one minimum at approximately the same location as the one shown in Fig. 7(a). Both MSE curves indicated the presence of only one heart sound. Nevertheless, only the results of the second heart sound are presented since larger correlation was obtained for this reference signal. Fig. 7(d) shows the cross-correlation result between the acquired respiratory signal in Fig. 6(c) and the manually selected second reference heart sound for this case as shown in Fig. 6(a). As can be seen from both the MSE curve and the cross-correlation result, the location of the heart sound component can easily be detected. The estimated time delay was 372 for both the JTDSE and the cross-correlation methods. The validity of this estimate can 
(a)

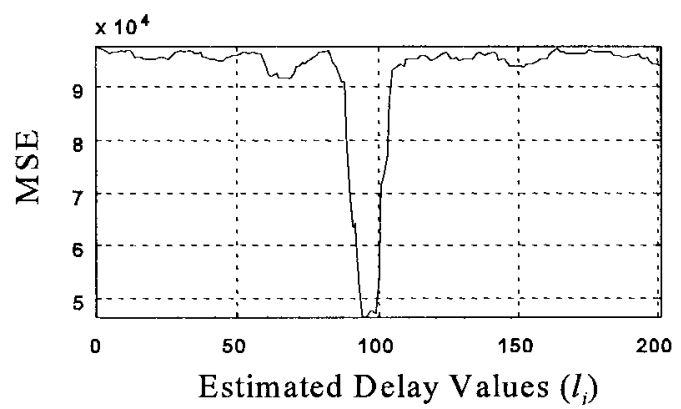

(b)

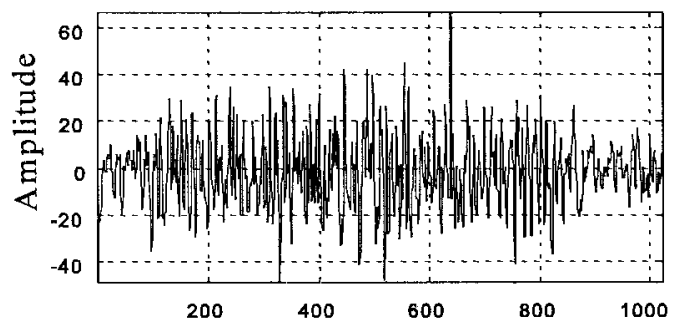

(c)

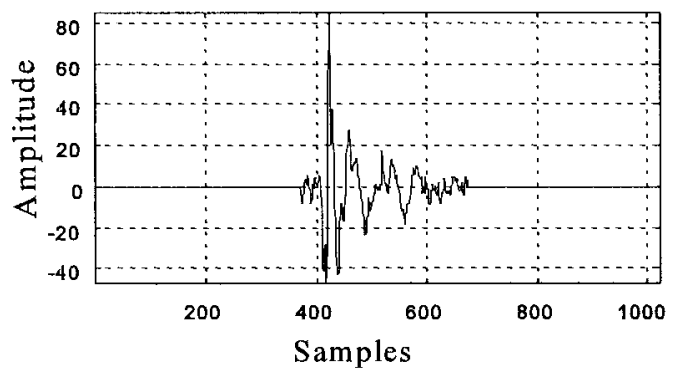

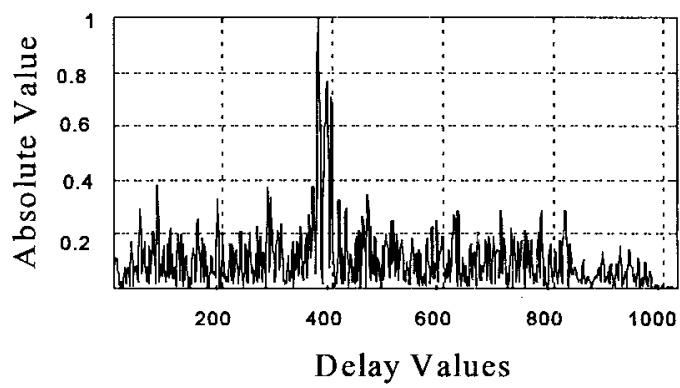

(d)

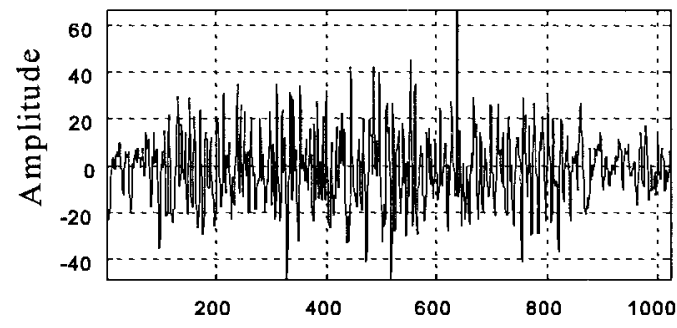

(e)

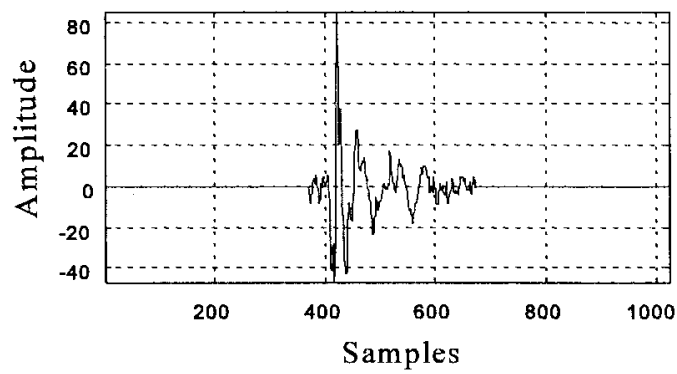

Fig. 7. Results for the healthy subject case. (a) MSE curve for the JTDSE for a second heart sound as the reference, (b) estimated respiratory signal for JTDSE, (c) estimated heart sound for JTDSE, (d) absolute value cross-correlation result between the manually-selected reference second heart sound and the acquired respiratory signal in Fig. 6(c), (e) estimated respiratory signal for cross-correlation method, and (f) estimated heart sound for cross-correlation method.

be approximately verified by visual inspection since the heart sound is prominent for this subject. Fig. 7(b), (e), (c), and (f) presents the estimated respiratory and heart sounds for these methods. As far as the estimation of the heart sounds components is concerned, this is considered to be a simple case since the heart sound is very prominent in the acquired respiratory section.

In contrast to the first case, the second case [as shown in Fig. 6(b) and (d], is complicated due to the factors mentioned earlier. In this case, due to low HRR, the order of the BFTF filter in the TDE was increased to 20. Fig. 8(a) shows the MSE curve obtained using the JTDSE method when the first heart sound was used as the reference signal for the JTDSE scheme. Again, the MSE curve presents well-defined and prominent minima at positions pointed out by A and B with estimated time delays at 936 and 2208, respectively. Fig. 9(a) shows the corresponding cross-correlation result between the signal section in Fig. 6(d) and the first heart sound reference selected for this case. The cross-correlation method provided timedelay estimates at 1000, 1329, and 2295. Next, the second heart sound was used as the reference signal for the JTDSE method. Fig. 8(b) presents the MSE curve for this case. Now the MSE curve presents two well-defined minima at positions pointed out by $\mathbf{C}$ and $\mathbf{D}$ with estimated time delays at 1312 and 2680. Points A, B, C, and $\mathbf{D}$ are indicated in the MSE curves in Fig. 8(a) and (b).

Considering the characteristics of these MSE curves it is observed that when a first heart sound was used as the reference, in Fig. 8(a) two prominent minima occurred at $\mathbf{A}$ and $\mathbf{B}$ indicating increased correlation at these positions, while the minima at positions $\mathbf{C}$ and $\mathbf{D}$ were not so prominent. On the other hand, when a second heart sound was used as the reference, Fig. 8(b) exhibits two prominent minima at positions indicated by $\mathbf{C}$ and $\mathbf{D}$, whereas, those at $\mathbf{A}$ and $\mathbf{B}$ are not so obvious for this reference. Taking into account the changes and similarities in the positions of the minima in these curves, one can conclude that two first heart sounds and two second heart sounds are present in the acquired respiratory section of Fig. 6(d). The first minimum, located around 60 in Fig. 8(a) and (b), is due to the sudden transition at the beginning of the acquired respiratory signal in Fig. 6(d) at approximately 500 . This transition might have been caused by a small movement of the microphone. Fig. 9(b) presents the result of the cross-correlation method for a second heart sound as the reference signal. This figure indicates the estimated time delay values at 1182 and 1369 . 
(a)

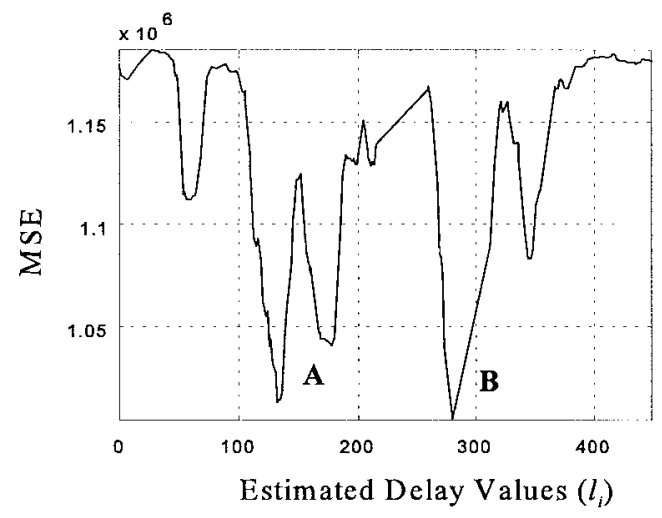

(c)

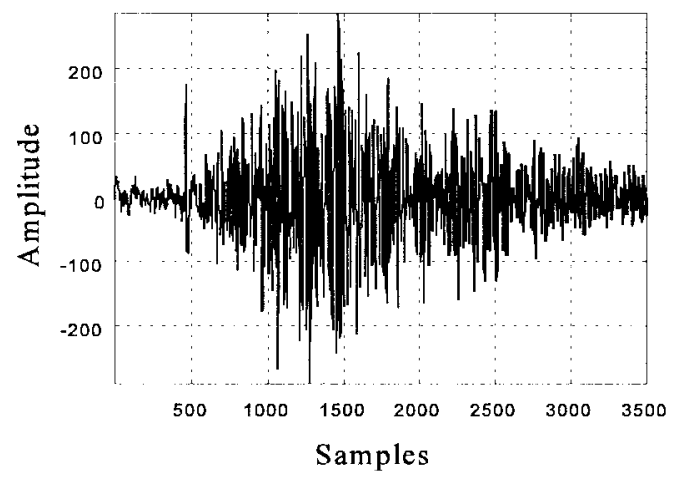

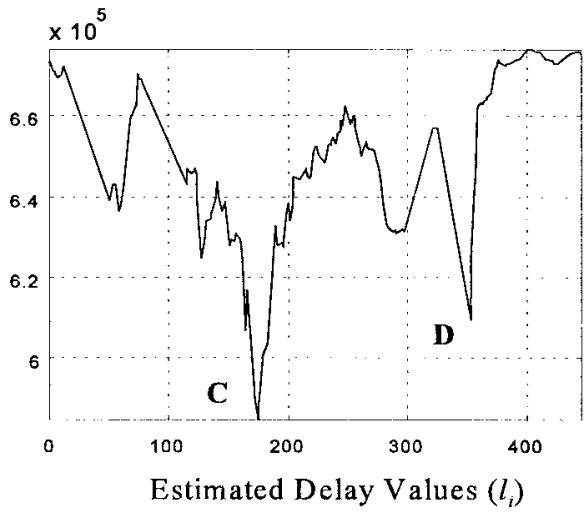

(b)

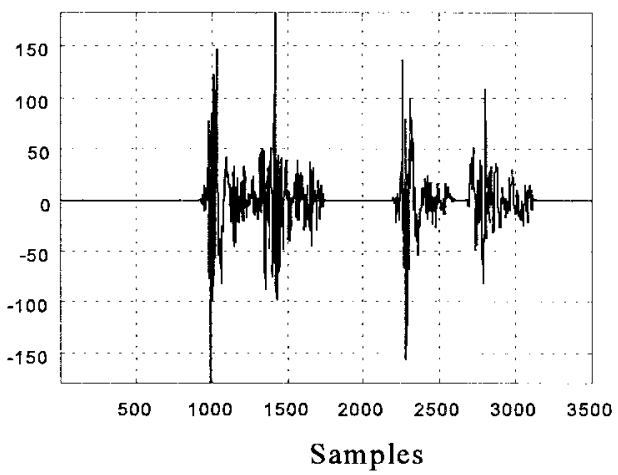

(d)

Fig. 8. Results for the asthmatic subject case in Fig. 6(d). (a) MSE curve for the JTDSE using a first heart sound as the reference, (b) MSE curve for the JTDSE using a second heart sound as the reference, (c) estimated respiratory signal, and (d) estimated interference heart sounds.

(a)

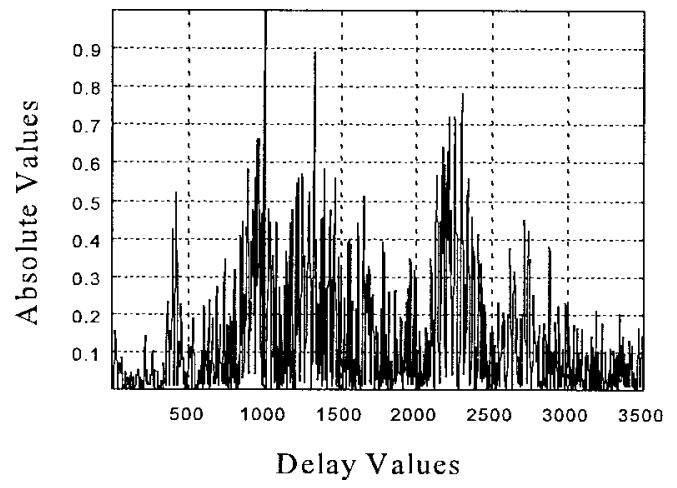

(c)

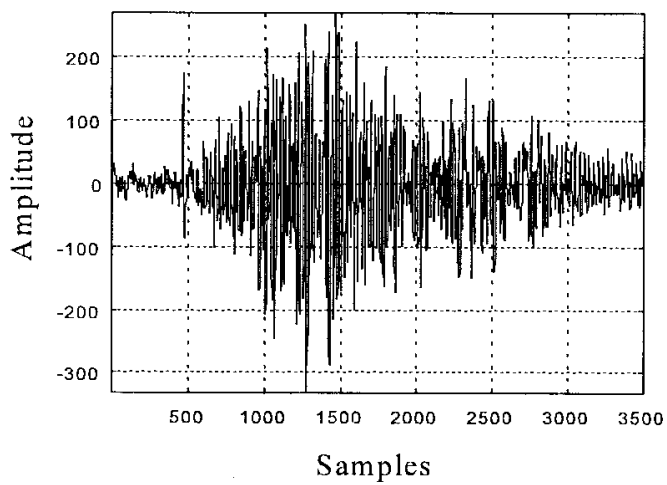

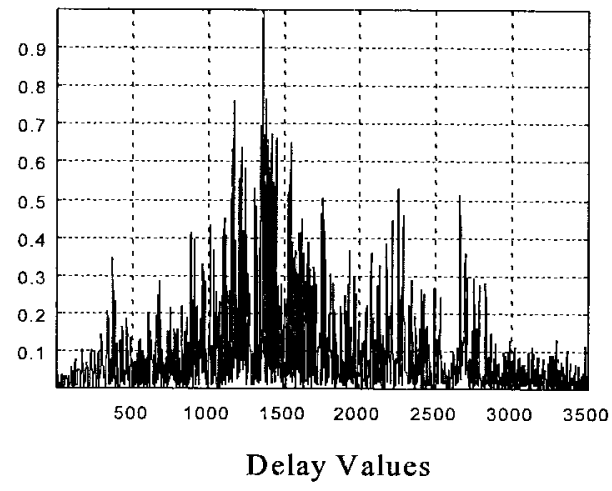

(b)

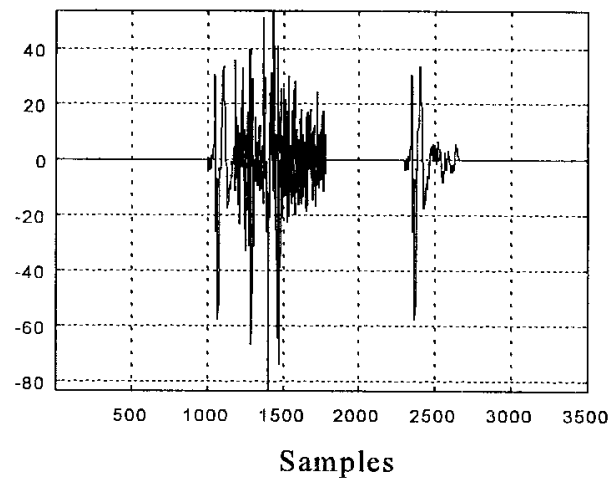

(d)

Fig. 9. Results for the asthmatic subject case. (a) Absolute value of the cross-correlation between a first reference heart sound from Fig. 6(b) and the acquired respiratory section of Fig. 6(d). (b) Absolute value of the cross-correlation between a second heart and the acquired respiratory section of Fig. 6(d). (c) Estimated respiratory signal and (d) estimated interference heart sounds. 
Once the time delays are estimated using both the JTDSE and cross-correlation method in conjunction with the adaptive filtering, a separate adaptive transversal filter of order eight is used in each subband to generate estimates of the heart and respiratory signals alone. Fig. 8(c) and (d) shows the estimated respiratory and heart sounds using the TDE results of the JTDSE scheme, respectively, while Fig. 9(c) and (d) shows these estimated signals based upon the results of the crosscorrelation method. As can be observed, in the latter case, the amplitudes of the heart sound estimates are decreased due to the fact that the cross-correlation method failed to determine the positions of the multiple heart sounds. The JTDSE method still provided accurate estimates of the positions of the multiple heart sound components in the acquired respiratory section even for this difficult case, hence, resulting in more reliable estimates of the respiratory and heart sounds.

\section{CONCLUSION}

In this paper, a new interference cancellation scheme is presented which provides estimates of the positions of the interference heart sounds signal and then separates these signals from the acquired respiratory signal. The main property of the JTDSE method is based upon the fact that the information about the time delay between reference and acquired signals is common to all the levels and information-bearing subbands. Consequently, this provides multiple "looks" of the signal at different levels/subbands. The method was tested on both synthesized and real respiratory signals. The performance of the JTDSE scheme was compared with the conventional adaptive filtering when used in conjunction with the cross-correlation method. The cross-correlation was used to provide the estimate of the time delays and a conventional adaptive filter was used for signal separation purposes. The results indicated that the proposed scheme is promising for applications that require simultaneous TDE and interference cancellation. The results on synthesized data showed that the proposed scheme is capable of estimating the time delays and their number very accurately, even under difficult conditions. The results on real data, on the other hand, showed the promise of the proposed scheme for real-life applications.

As always, there is a tradeoff between the computational complexity of the algorithm and the accuracy of the TDE. If a signal has a length $L$ and the adaptive filter order used in the JTDSE scheme is $M$, the order of operations per delay adaptation according to 11(a) is $O(L M)$, for each subband at the third level of decomposition. In contrast, the order of operations per delay is $O(L)$ for the crosscorrelation method. Even though the proposed JTDSE scheme is computationally more demanding than the cross-correlation method, the JTDSE offers substantially better and more reliable time-delay estimates. In addition, an optimum number of subbands and levels can be determined using wavelet packets [20], [21] to maximize the entropy measure in those bands. This optimal subband decomposition not only reduces the computational time, but also permits the decomposition of the time-scale plane best adapted to the signal characteristics. The JTDSE method can effectively be used in areas such as target detection, direction of arrival estimation, and equalization.

\section{REFERENCES}

[1] A. Jones, "A brief overview of the analysis of respiratory sounds," Physioteraphy, vol. 81, no. 1, pp. 41-57, Jan. 1995.

[2] M. J. Mussell, "The need for standards in recording and analysis of respiratory sounds," Med. Biol. Eng., Comput., vol. 30, pp. 129-139, 1992.

[3] Y. Ploysongsang, V. Iyer, and P. Ramamoorthy, "Reproducibility of vesicular breath sounds in normal subjects," Respir., vol. 58, pp. 158-162, 1991.

[4] N. Gavriely, Y. Palti, and G. Alroy, "Spectral characteristics of normal breath sounds," J. Appl. Physiol., vol. 50, pp. 307-314, 1981.

[5] S. W. Banham, R. B. Urquhart, J. E. S. Macleod, and F. Moran, "Alteration in the low-frequency lung sounds in respiratory disorders associated with crackles," Eur. J. Respir. Dis., vol. 65, pp. 58-63, 1984.

[6] V. K. Iyer, P. Ramamoorthy, H. Fan, and Y. Ploysongsang, "Reduction of heart sounds from lung sounds by adaptive filtering," IEEE Trans. Biomed. Eng., vol. 33, no. 12, pp. 1141-1148, 1986.

[7] M. Kompis and E. Russi "Adaptive heart-noise reduction of lung sounds recorded by a single microphone," in Proc. 14th Annu. Int. Conf. IEEE Eng. Med., Biol. Soc., 1992, pp. 691-692.

[8] G. Sod-Moriah, O. Gelber, G. Gurman, and A. Cohen "On line ventilation monitoring during anesthesia and respiratory intensive care," in Proc. 18th Convention Electrical and Electronics Engineers, 1995, p. 4.2-2.

[9] S. Charleston and M. R. Azimi-Sadjadi, "Reduced-order Kalman filtering for the enhancement of respiratory sounds," IEEE Trans. Biomed. Eng., vol. 43, no. 4, pp. 421-424, Apr. 1996; vol. 43, no. 6, p. 668.

[10] B. Harris, I. Gath, G. Rondouin, and C. Feuerstein, "On time-delay estimation of epileptic EEG," IEEE Trans. Biomed. Eng., vol. 41, no. 9 , pp. 820-829, 1994.

[11] A. H. Quazi, "An overview on the time delay estimate in active and passive systems for target localization," IEEE Trans. Acoust., Speech, Signal Processing, vol. 29, no. 3, pp. 527-533, June 1981.

[12] I. Daubechies, Ten Lectures on Wavelets, CBMS-NSF Series in Appl. Math. Philadelphia, PA: SIAM, 1992.

[13] S. G. Mallat, "A theory for multiresolution signal decomposition: The wavelet representation," IEEE Trans. Pattern Anal. Machine Intell., vol. 11, no. 7, pp. 674-693, July 1989.

[14] M. Vetterli, "Wavelets and filter banks: theory and design," IEEE Trans. Signal Processing, vol. 40, no. 9, pp. 2207-2232, Sept. 1992

[15] T. R. Cuthbert, Optimization Using Personal Computers. New York: Wiley-Interscience 1987.

[16] J. M. Cioffi, "The block-processing FTF adaptive algorithm," IEEE Trans. Acoust., Speech, Signal Processing, vol. ASSP-34, no. 1, pp. 77-90, Feb. 1986.

[17] Y. T. Chan, J. M. Riley, and J. B. Plant, "A parameter estimation approach to time-delay estimation and signal detection," IEEE Trans. Acoust., Speech, Signal Processing, vol. ASSP-28, no. 1, pp. 8-15, Feb. 1980.

[18] A. V. Oppenheim and R. W. Schafer, Digital Signal Processing. Englewood Cliffs, NJ: Prentice-Hall, 1989.

[19] L. E. Baker, "Applications of the impedance technique to the respiratory system," IEEE Eng. Med. Biol. Mag., vol. 8, no. 1, pp. 50-52, Mar. 1989.

[20] C. B. Lambrech and M. Karrakchou, "Sub-band adaptive filtering: the mutual wavelet packets approach," in Proc. ICASSP'93, Apr. 1993, vol. III, pp. 316-319.

[21] R. R. Coifman and M. W. Wickerhauser, "Entroy-based algorithms for best basis selection," IEEE Trans. Inform. Theory, vol. 38, pp. 713-718, Mar. 1992.

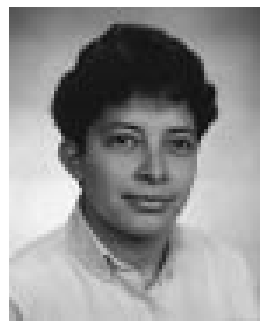

Sonia Charleston was born in Oaxaca, Mexico, in 1960. She received the M.S. degree in biomedical engineering in 1987 and the Ph.D. degree in sciences in 1996, both from the Universidad Autónoma Metropolitana of Mexico, Mexico City, Mexico While studying for the Ph.D. degree, she was involved in digital signal/image processing research in the Department of Electrical Engineering at Colorado State University, Fort Collins.

Currently, she is an Associate Professor at the Universidad Autónoma Metropolitana. Her interests are related to digital signal and image processing as applied to biomedical problems. 


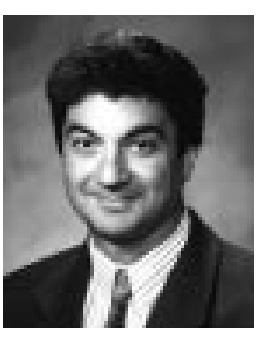

Mahmood R. Azimi-Sadjadi (S'81-M81-SM'89) was born in Tehran, Iran, in 1952. He received the B.S. degree from University of Tehran, Iran, in 1977 and the M.Sc. and Ph.D. degrees from the Imperial College, University of London, U.K., in 1978 and 1982 respectively, all in electrical engineering.

He served as an Assistant Professor in the Department of Electrical and Computer Engineering, University of Michigan, Dearborn. Since July 1986 he has been with the Department of Electrical Engineering, Colorado State University, Fort Collins, where he is now a Professor. Currently, he is the Director of the Multisensory Computing Laboratory (MUSCL) at Colorado State University. His areas of interest are digital signal/image processing, target detection and tracking, multidimensional system theory and analysis, adaptive filtering, system identification, and neural networks. His contributions in these areas resulted in more than 100 journals and refereed conference publications. He is a co-author of Digital Filtering in One and Two Dimensions (New York: Plenum, 1989).

Dr. Azimi-Sadjadi is the recipient of 1993 ASEE-Navy Senior Faculty Fellowship Award, 1991 CSU Dean's Council Award, 1990 Battelle Summer Faculty Fellowship Award, and the 1984 DOW chemical Outstanding Young Faculty Award of the American Society for Engineering Education. He is an Associate Editor of the IEEE Transactions on Signal Processing.

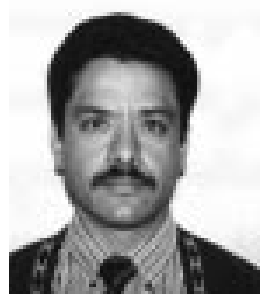

Ramon González-Camarena was born in Mexico City, Mexico, where he received the M.D. degree from the Autonomous National University of Mexico (UNAM) in 1978

He has been a Professor of Physiological Sciences with pre- and postgraduate students of the Biomedical Engineering and Experimental Biology disciplines since 1978 and has been an Investigator in the Medical Research Area of the Health Science Department, at the Metropolitan Autonomous University, campus Iztapalapa (UAM-I). His research interests are in the general areas of cardiopulmonary physiology, lung sounds, heart rate variability, and biomedical signal processing. 